\section{(2) OPEN ACCESS}

\title{
Sex differences in investigations and outcomes among patients with type 2 myocardial infarction
}

\author{
Dorien M Kimenai (D) , 1 Bertil Lindahl, ${ }^{2,3}$ Andrew R Chapman (D) , ${ }^{4}$ Tomasz Baron, ${ }^{3}$ \\ Anton Gard, ${ }^{3}$ Ryan Wereski, ${ }^{4}$ Steven J R Meex, ${ }^{5,6}$ Tomas Jernberg, ${ }^{7}$ \\ Nicholas L Mills (D) , ${ }^{1,4}$ Kai M Eggers ${ }^{3}$
}

- Additional supplemental material is published online only. To view, please visit the journal online (http://dx.doi. org/10.1136/heartjnl-2021319118).

${ }^{1}$ Usher Institute, University of Edinburgh, Edinburgh, UK ${ }^{2}$ Uppsala Clinical Research Center, Uppsala University, Uppsala, Sweden

${ }^{3}$ Department of Medical Sciences, Cardiology, Uppsala University, Uppsala, Sweden ${ }^{4}$ BHF Centre for Cardiovascular Science, University of Edinburgh, Edinburgh, UK

${ }^{5}$ Central Diagnostic Laboratory, Maastricht University Medical Center, Maastricht, Netherlands ${ }^{6}$ CARIM School for Cardiovascular Diseases, Maastricht University, Maastricht, Netherlands ${ }^{7}$ Department of Clinical Sciences, Danderyd University Hospital, Karolinska Institutet, Stockholm, Sweden

\section{Correspondence to} Dr Dorien M Kimenai, Usher Institute, University of Edinburgh, Edinburgh EH16 4SA, UK;

dorien.kimenai@ed.ac.uk

Received 2 February 2021

Revised 1 April 2021

Accepted 4 April 2021

\begin{abstract}
Objectives Type 2 myocardial infarction (MI) is a heterogenous condition and whether there are differences between women and men is unknown. We evaluated sex differences in clinical characteristics, investigations and outcomes in patients with type $2 \mathrm{Ml}$. Methods In the Swedish Web based system for Enhancement and Development of Evidence based care in Heart disease Evaluated According to Recommended Therapies (SWEDEHEART) registry, we compared patients admitted to coronary care units with a diagnosis of type 1 or type 2 Ml. Sex-stratified Cox regression models evaluated the association with all-cause death in men and women separately.
\end{abstract}

Results We included 57264 (median age 73 years, $65 \%$ men) and 6485 (median age 78 years, 50\% men) patients with type 1 and type $2 \mathrm{Ml}$, respectively. No differences were observed in the proportion of men and women with type $2 \mathrm{Ml}$ who underwent echocardiography and coronary angiography, but women were less likely than men to have left ventricular (LV) impairment and obstructive coronary artery disease (CAD). Compared with type $1 \mathrm{Ml}$, patients with type $2 \mathrm{Ml}$ had higher risk of death regardless of sex (men: adjusted HR $1.55(95 \% \mathrm{Cl}$ 1.44 to 1.67); women: adjusted HR $1.34(95 \% \mathrm{Cl} 1.24$ to 1.45$)$ ). In those with type $2 \mathrm{MI}$, the risk of death was lower for women than men (adjusted HR $0.85(95 \% \mathrm{Cl}$ 0.76 to 0.92 ) (men, reference)).

Conclusions Type $2 \mathrm{Ml}$ occurred in men and women equally and we found no evidence of sex bias in the selection of patients for cardiac investigations. Patients with type $2 \mathrm{Ml}$ had worse outcomes, but women were less likely to have obstructive CAD or severe LV impairment and were more likely to survive than men.

\section{INTRODUCTION}

Patients with type 2 myocardial infarction (MI) have a poor prognosis, ${ }^{12}$ and this condition is a heterogeneous entity with multiple aetiologies and triggers. ${ }^{3}$ Insights into the patient groups who are at highest risk and the clinical features that influence outcomes are required to develop evidence-based treatments that reduce mortality.

It has been observed that the majority of patients diagnosed with type $1 \mathrm{MI}$ are men. Intriguingly, recent studies have suggested that the proportion of patients with type 2 MI who are women is higher. ${ }^{4-7}$ Similarly, the investigation and treatment of type 1 MI differs in men and women, with women less likely to receive evidence-based therapies. ${ }^{8-10}$ Whether the clinical characteristics and investigations differ in men and women with type $2 \mathrm{MI}$, and how these impact on outcomes is not known.

Here, we compare the clinical characteristics, investigations and outcomes of consecutive patients with type 1 and type 2 MI stratified by sex. Our aim was to define the predictors of death and major adverse cardiovascular events (MACE) in patients with a diagnosis of type $2 \mathrm{MI}$, and to determine whether these differ in men and women.

\section{METHODS}

\section{Study population}

Patients were enrolled in the Swedish Web based system for Enhancement and Development of Evidence based care in Heart disease Evaluated According to Recommended Therapies (SWEDEHEART) registry between September 2010 and May 2018. The rationale and methods for data collection have been described previously. ${ }^{11}$ The SWEDEHEART registry includes consecutive patients admitted to coronary care units (CCU) or other specialised facilities at all Swedish hospitals. To ensure the correctness and quality of the data entered, hospitals are monitored on a regular basis. ${ }^{11}$ Patients included in the registry are informed about their participation which they have the right to decline. Written consent is not required according to Swedish law.

For this study, we included all unique patients with a diagnosis of type 1 or type $2 \mathrm{MI}$ according to the treating physician based on the criteria outlined in the Universal Definition of Myocardial Infarction. ${ }^{12}$ To standardise the study cohort, we included only patients with available maximum results for highsensitivity cardiac troponin T (hs-cTnT), and did not consider patients assessed at sites using other cardiac troponin assays. Similarly, we did not consider patients with ST-elevation MI (STEMI) in the primary analysis as care pathways for this condition are highly standardised and the majority of patients have type 1 MI. Patients with STEMI were, however, included in a secondary analysis to verify the robustness of our findings across all patients with MI. The present manuscript follows the Strengthening the Reporting of Observational Studies in Epidemiology guidelines. ${ }^{13}$

\section{Patient and public involvement}

This research was done without patient involvement. 


\section{Clinical outcomes}

We used the Swedish Patient Registry (hospitalisation dates and discharge diagnoses based on International Classification of Diseases, 10th revision (ICD-10) codes) and the Swedish Population Registry to obtain clinical outcomes. The accuracy of hospital discharge diagnosis codes in this registry has previously been evaluated showing $85 \%$ to $95 \%$ validity. ${ }^{14}$ We investigated the occurrence of all-cause death and MACE until May 2018 and December 2017 respectively. MACE was defined as a composite end point of all-cause mortality, readmissions for non-fatal MI

Table 1 Clinical characteristics of patients with type 1 myocardial infarction and type 2 myocardial infarction, stratified by sex

\begin{tabular}{|c|c|c|c|c|c|c|}
\hline \multirow[b]{2}{*}{ Clinical characteristics* } & \multicolumn{3}{|c|}{ Type 1 myocardial infarction } & \multicolumn{3}{|c|}{ Type 2 myocardial infarction } \\
\hline & $\begin{array}{l}\text { All } \\
(n=57264)\end{array}$ & $\begin{array}{l}\text { Men } \\
(n=37 \text { 397) }\end{array}$ & $\begin{array}{l}\text { Women } \\
(n=19867)\end{array}$ & $\begin{array}{l}\text { All } \\
(n=6485)\end{array}$ & $\begin{array}{l}\text { Men } \\
(n=3232)\end{array}$ & $\begin{array}{l}\text { Women } \\
(n=3253)\end{array}$ \\
\hline \multicolumn{7}{|l|}{ Demographics } \\
\hline Age (years) & $73(64-81)$ & $71(62-79)$ & $77(68-84)$ & $78(70-85)$ & $77(69-84)$ & $80(71-86)$ \\
\hline Body mass index $\left(\mathrm{kg} / \mathrm{m}^{2}\right)$ & $26.6(24.1-29.7)$ & $26.8(24.5-29.7)$ & $26.2(23.1-29.8)$ & $25.6(22.9-29.1)$ & $25.9(23.5-28.7)$ & $25.4(22.3-29.3)$ \\
\hline \multicolumn{7}{|l|}{ Risk factors } \\
\hline Current smoking & $9636(18 \%)$ & $6528(18 \%)$ & $3108(17 \%)$ & $809(14 \%)$ & $410(15 \%)$ & $399(14 \%)$ \\
\hline Hypertension & 33313 (59\%) & $20592(55 \%)$ & $12721(64 \%)$ & $4015(62 \%)$ & $1977(62 \%)$ & $2038(63 \%)$ \\
\hline Diabetes & $15152(27 \%)$ & $9717(26 \%)$ & $5435(27 \%)$ & $1889(29 \%)$ & $1056(33 \%)$ & $833(26 \%)$ \\
\hline Hyperlipidaemia & $22520(40 \%)$ & $15189(41 \%)$ & $7331(37 \%)$ & $2892(45 \%)$ & $1636(51 \%)$ & $1256(39 \%)$ \\
\hline eGFR (CKD-EPI, mL/kg/m²) & $73(52-88)$ & $75(56-90)$ & $66(47-84)$ & $57(37-79)$ & $57(36-78)$ & $58(38-79)$ \\
\hline \multicolumn{7}{|l|}{ Medical history } \\
\hline Previous myocardial infarction & $18868(33 \%)$ & $12555(34 \%)$ & $6313(32 \%)$ & $2553(40 \%)$ & $1437(45 \%)$ & $1116(35 \%)$ \\
\hline Previous CABG & $7155(13 \%)$ & $5399(15 \%)$ & $1756(9 \%)$ & $989(15 \%)$ & $675(21 \%)$ & $314(10 \%)$ \\
\hline Previous PCI & $12711(22 \%)$ & $8996(24 \%)$ & $3715(19 \%)$ & $1279(20 \%)$ & $804(25 \%)$ & $475(15 \%)$ \\
\hline Heart failure & $7014(13 \%)$ & $4632(13 \%)$ & $2382(12 \%)$ & $1282(21 \%)$ & $764(25 \%)$ & $518(17 \%)$ \\
\hline Previous stroke & $5401(10 \%)$ & $3416(9 \%)$ & $1985(10 \%)$ & $870(14 \%)$ & $490(15 \%)$ & $380(12 \%)$ \\
\hline COPD & $4833(8 \%)$ & $2716(7 \%)$ & $2117(11 \%)$ & $1158(18 \%)$ & $527(16 \%)$ & $631(19 \%)$ \\
\hline Dementia & $440(1 \%)$ & $251(1 \%)$ & $189(1 \%)$ & $80(1 \%)$ & $36(1 \%)$ & $44(1 \%)$ \\
\hline Previous or present cancer & $2410(4 \%)$ & $1779(5 \%)$ & $631(3 \%)$ & $497(8 \%)$ & $351(11 \%)$ & $146(5 \%)$ \\
\hline \multicolumn{7}{|l|}{ ECG findings } \\
\hline \multicolumn{7}{|l|}{ Heart rhythm } \\
\hline Sinus rhythm & $49574(87 \%)$ & $32382(87 \%)$ & $17192(87 \%)$ & $4354(68 \%)$ & $2075(65 \%)$ & $2279(71 \%)$ \\
\hline Atrial fibrillation/flutter & $5798(10 \%)$ & $3691(10 \%)$ & $2107(11 \%)$ & $1756(27 \%)$ & $909(28 \%)$ & $847(26 \%)$ \\
\hline Other & $1512(3 \%)$ & $1057(3 \%)$ & $455(2 \%)$ & $322(5 \%)$ & $218(7 \%)$ & $104(3 \%)$ \\
\hline \multicolumn{7}{|l|}{ Ischaemic findings } \\
\hline ST-segment depression & $17773(31 \%)$ & $11373(31 \%)$ & $6400(33 \%)$ & $2211(35 \%)$ & $1148(36 \%)$ & $1063(33 \%)$ \\
\hline T-wave inversion & $7507(13 \%)$ & $4723(13 \%)$ & $2784(14 \%)$ & $637(10 \%)$ & $285(9 \%)$ & $352(11 \%)$ \\
\hline Other ST-segment changes & $12832(23 \%)$ & $8299(22 \%)$ & $4533(23 \%)$ & $1725(27 \%)$ & $928(30 \%)$ & $797(25 \%)$ \\
\hline No ST-segment changes & $18489(33 \%)$ & $12567(34 \%)$ & $5922(30 \%)$ & $1768(28 \%)$ & $799(25 \%)$ & $969(31 \%)$ \\
\hline \multicolumn{7}{|l|}{ In-hospital examinations and intervention } \\
\hline Echocardiography & $44668(78 \%)$ & $29877(80 \%)$ & $14791(75 \%)$ & $4201(65 \%)$ & $2103(65 \%)$ & $2098(65 \%)$ \\
\hline Coronary angiography & $45229(79 \%)$ & $31184(83 \%)$ & $14045(71 \%)$ & $2272(35 \%)$ & $1150(36 \%)$ & $1122(35 \%)$ \\
\hline $\mathrm{PCl}$ & $33083(58 \%)$ & $23592(63 \%)$ & $9491(48 \%)$ & $734(11 \%)$ & $463(14 \%)$ & $271(8 \%)$ \\
\hline CABG & $4069(7 \%)$ & $3163(9 \%)$ & $906(5 \%)$ & $76(1 \%)$ & $63(2 \%)$ & $13(0 \%)$ \\
\hline \multicolumn{7}{|l|}{ Angiographic findings } \\
\hline Inconclusive & $79(0 \%)$ & $48(0 \%)$ & $31(0 \%)$ & $3(0 \%)$ & $2(0 \%)$ & $1(0 \%)$ \\
\hline Normal or non-occlusive disease & $4562(11 \%)$ & $1942(7 \%)$ & $2620(19 \%)$ & $1138(50 \%)$ & $385(33 \%)$ & $753(66 \%)$ \\
\hline 1-vessel to 2-vessel obstructive disease & $25024(57 \%)$ & $17465(58 \%)$ & $7559(56 \%)$ & $656(28 \%)$ & $392(34 \%)$ & $264(23 \%)$ \\
\hline 3-vessel obstructive disease/left main stem & $13858(32 \%)$ & $10412(35 \%)$ & $3446(25 \%)$ & $499(22 \%)$ & $376(33 \%)$ & $123(11 \%)$ \\
\hline \multicolumn{7}{|l|}{ Left ventricular ejection fraction } \\
\hline$\geq 50 \%$ & $28938(65 \%)$ & $19341(65 \%)$ & $9597(65 \%)$ & $2319(56 \%)$ & $1056(51 \%)$ & $1263(61 \%)$ \\
\hline $31 \%-49 \%$ & $13158(30 \%)$ & $8762(29 \%)$ & $4396(30 \%)$ & $1483(35 \%)$ & $805(38 \%)$ & $678(32 \%)$ \\
\hline$\leq 30 \%$ & $2361(5 \%)$ & $1633(6 \%)$ & $728(5 \%)$ & $376(9 \%)$ & $229(11 \%)$ & $147(7 \%)$ \\
\hline \multicolumn{7}{|l|}{ Medication at discharge } \\
\hline Aspirin & $51547(91 \%)$ & $34126(92.4 \%)$ & $17421(89.1 \%)$ & $4135(65 \%)$ & $2110(67 \%)$ & $2025(64 \%)$ \\
\hline P2Y12 inhibitors & $45751(81 \%)$ & $30334(82 \%)$ & $15417(79 \%)$ & $2305(36 \%)$ & $1260(40 \%)$ & $1045(33 \%)$ \\
\hline Anticoagulants & $5319(9 \%)$ & $3481(9 \%)$ & $1838(9 \%)$ & $1186(19 \%)$ & $583(18 \%)$ & $603(19 \%)$ \\
\hline Beta-blockers & $48851(87 \%)$ & $32022(87 \%)$ & $16829(86 \%)$ & $5056(80 \%)$ & $2555(81 \%)$ & $2501(78 \%)$ \\
\hline ACEI & $30450(54 \%)$ & $21061(57 \%)$ & $9389(48 \%)$ & $2628(41 \%)$ & $1401(44 \%)$ & $1227(38 \%)$ \\
\hline ARB & $13106(23 \%)$ & $7995(22 \%)$ & $5111(26 \%)$ & $1419(22 \%)$ & $645(20 \%)$ & $774(24 \%)$ \\
\hline Statins & $49947(88 \%)$ & $33811(92 \%)$ & $16136(83 \%)$ & $4084(64 \%)$ & $2206(70 \%)$ & $1878(59 \%)$ \\
\hline hs-cTnT (ng/L) & $269(100-782)$ & $280(100-843)$ & $250(98-684)$ & $272(115-666)$ & $298(121-804)$ & $246(107-570)$ \\
\hline Duration of hospital stays (days) & $4(3-6)$ & $4(3-7)$ & $4(3-7)$ & $5(3-8)$ & $5(3-8)$ & $5(3-8)$ \\
\hline
\end{tabular}

Continuous data are presented as mean (SD) or mean (IQR), as appropriate. Categorical data are presented as $\mathrm{n}(\%)$.

${ }^{*}$ The number $(\%)$ of missing values of clinical characteristics are presented in online supplemental table 1.

ACEl, ACE inhibitor; ARB, angiotensin II receptor blockers; CABG, coronary artery bypass graft; CKD-EPI, Chronic Kidney Disease Epidemiology Collaboration; COPD, chronic obstructive pulmonary disease; eGFR, estimated glomerular filtration rate; hs-cTnT, high-sensitivity cardiac troponin T; PCl, percutaneous coronary intervention. 


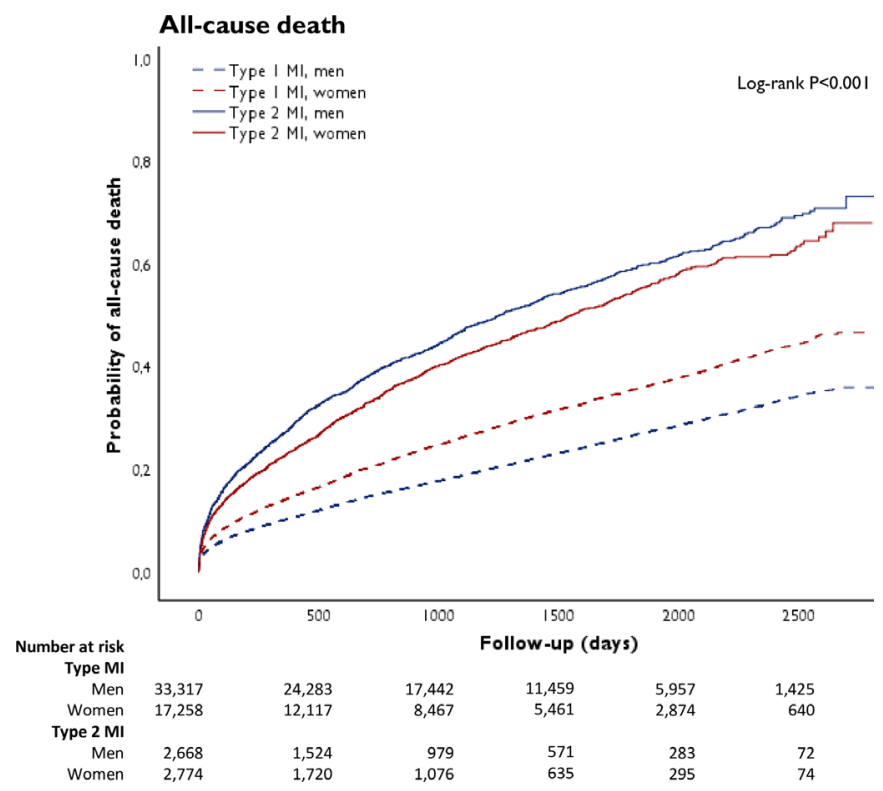

Figure 1 Crude cumulative occurrence of all-cause death in patients with type 1 and type 2 myocardial infarction (MI), stratified by sex.

(I21), heart failure (I50) and ischaemic stroke (I63). During the first 30 days after the index hospitalisation, it was not possible to separate a new MI from an index MI, and therefore only MI occurring 30 days after the index hospitalisation was included.

\section{Statistical analysis}

Comparisons were made between men and women, and between those with type 1 and type 2 MI. Sex-specific incidence rates were determined and Kaplan-Meier curves were constructed to investigate the occurrence of all-cause death and MACE over time. Univariable and multivariable cox regression analyses were performed to investigate differences between groups (type 1 $\mathrm{MI}=$ reference; $\mathrm{men}=$ reference) on clinical outcomes using three models with sequential adjustment of covariates. Model 1 presents the univariable model (model 1: crude model+hospital site, admission year). We were interested whether the differences would be attenuated when we adjusted for comorbidities, which is presented in model 2 (model 2: model 1+adjusted for age, current smoking, hypertension, diabetes, hyperlipidaemia, coronary artery disease (CAD, defined as previous MI or coronary revascularisation procedure), congestive heart failure, previous stroke, ST-segment depression, estimated glomerular filtration rate (eGFR), chronic obstructive pulmonary disease (COPD), dementia, previous or present cancer). Model 3 was additionally adjusted for hs-cTnT concentrations since sex-specific variations in the extent of myocardial damage potentially might have affected our findings (model 3: model 2+hs-cTnT concentrations). To obtain a normal distribution, we naturally log transformed eGFR and hs-cTnT before they entered the model. To investigate the relationship between clinical characteristics and outcomes in men and women with type 2 MI, we conducted multivariable Cox regression analysis. We tested for interactions between these variables and sex and a $\mathrm{p}$ value $<0.1$ was considered to be statistically significant. Due to the low proportion of missing values of variables (online supplemental table 1), no imputation techniques were conducted. In secondary analyses, we (1) included patients with STEMI and (2) included only patients who underwent coronary angiography (CAG), and we adjusted additionally for the extent of CAD (categorised as normal or non-occlusive disease (reference), 1-vessel to 2-vessel obstructive disease, 3-vessel obstructive disease/left main stem and inconclusive findings). Statistical analysis was done by SPSS V.26.0 (IBM).

\section{RESULTS}

After excluding patients with a diagnosis other than type 1 or type $2 \mathrm{MI}$ and those without hs-cTnT results, 63749 hospital admissions were included in our study (online supplemental figure 1). Of these, 57264 and 6485 were diagnosed with type 1 and type $2 \mathrm{MI}$, respectively. More men than women were diagnosed with type 1 MI (37 397/57 264 (65\%) vs 19 867/57 264 $(35 \%))$, whereas the proportion of men and women with type 2 MI was the same (3232/6485 (50\%) vs 3253/6485 (50\%)).

\section{Clinical characteristics}

Patients with type $2 \mathrm{MI}$ were older and had a lower eGFR compared with those with type 1 MI, but they had a similar prevalence of risk factors and comorbidities (table 1). Men with type 2 MI were on average 3 years younger than women. They were more likely to have diabetes mellitus and hyperlipidaemia. Men more often had a prior history of MI, coronary revascularisation and heart failure. Peak hs-cTnT levels were higher for men than women.

\section{In-hospital investigations}

Patients with type $2 \mathrm{MI}$ were less likely than those with type $1 \mathrm{MI}$ to undergo echocardiography, CAG or revascularisation. In patients with type $2 \mathrm{MI}$, no difference was observed in the proportion of men and women who underwent echocardiography. However, in those who were scanned, a normal ejection fraction was observed less often in men than women (51\% vs 61\%). Likewise, a similar proportion of men and women with type $2 \mathrm{MI}$ underwent CAG. Women were twice as likely to have normal coronary arteries or non-obstructive cardiac disease than men (66\% vs 33\%), and therefore were less likely to undergo coronary revascularisation (9\% vs 16\%). Prescription of medications at discharge tended to

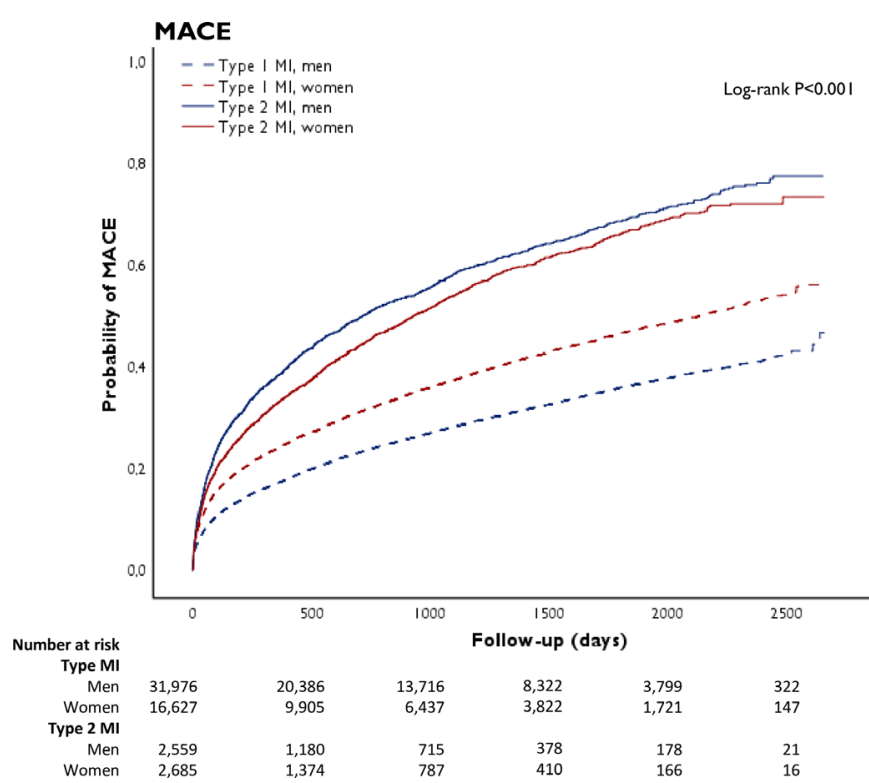

Figure 2 Crude cumulative occurrence of major adverse cardiovascular events (MACE) in patients with type 1 and type 2 myocardial infarction (MI), stratified by sex. MACE is defined as a composite end point of all-cause mortality, readmissions for non-fatal $\mathrm{Ml}$, heart failure and ischaemic stroke. 
Table 2 Incidence of all-cause death and major adverse events in women and men with type 2 or type 1 myocardial infarction

\begin{tabular}{|c|c|c|c|c|}
\hline & \multicolumn{2}{|l|}{ Men $(n=35985)$} & \multicolumn{2}{|l|}{ Women ( $n=20032)$} \\
\hline & N (\%) & Incidence rate & N (\%) & Incidence rate \\
\hline \multicolumn{5}{|l|}{ Type 1 myocardial infarction* } \\
\hline All-cause death & $7147 / 33317(22 \%)$ & $69 / 1000$ person-years & $5064 / 17258(29 \%)$ & $99 / 1000$ person-years \\
\hline Major adverse cardiovascular events & 9655/31 $977(30 \%)$ & $116 / 1000$ person-years & $6419 / 16628(39 \%)$ & $160 / 1000$ person-years \\
\hline \multicolumn{5}{|l|}{ Type 2 myocardial infarctiont } \\
\hline All-cause death & $1334 / 2668(50 \%)$ & $215 / 1000$ person-years & $1245 / 2774(45 \%)$ & $183 / 1000$ person-years \\
\hline Major adverse cardiovascular events & $1490 / 2559(58 \%)$ & $316 / 1000$ person-years & $1435 / 2685(53 \%)$ & $275 / 1000$ person-years \\
\hline
\end{tabular}

*Type 1 myocardial infarction no follow-up data available for major adverse cardiovascular events, $\mathrm{n}=1970(3.9 \%)$.

tType 2 myocardial infarction no follow-up data available for major adverse cardiovascular events, $\mathrm{n}=198$ (3.6\%).

be higher for men than women, particularly the prescription of statins was higher in men. In an exploratory analysis, we observed no association with female sex and the prescription of statins when accounting for comorbidities and angiographic findings (adjusted OR 0.84 (95\% CI 0.67 to 1.05$)$ ).

\section{Clinical outcomes}

A total of 56017 unique patients were identified of whom 50575 and 5442 had a diagnosis of type 1 and type 2 MI, respectively (online supplemental figure S1). These patients were included in the prognostic analyses (figures 1 and 2). For all-cause death, the median follow-up was $2.8(1.2-4.8)$ and 2.6 (1.0-4.6) years for men and women, respectively. For MACE, the median follow-up was $2.1(0.7-4.1)$ and $1.9(0.5-3.8)$ years for men and women, respectively. During follow-up, 22\% (7147/33 317) of men and $29 \%(5064 / 17238)$ of women with type $1 \mathrm{MI}$ died, compared with $50 \%(1334 / 2668)$ of men and $45 \%(1245 / 2774)$ of women with type 2 MI (table 2). MACE occurred in 30\% (9655/31 977) of men and 39\% (6419/16 628) of women with type $1 \mathrm{MI}$, compared with $58 \%(1490 / 2559)$ of men and 53\% $(1435 / 2,685)$ of women with type $2 \mathrm{MI}$.

We compared the crude risk of all-cause death and MACE between patients with type 1 and type $2 \mathrm{MI}$; men with type $2 \mathrm{MI}$ were at highest risk, while men with type $1 \mathrm{MI}$ were at lowest risk for both outcomes. For both men and women with type $2 \mathrm{MI}$, the crude risk of death was higher compared with type 1 MI. These differences were attenuated but remained significant after adjustment for risk factors and comorbidities (men: HR 1.54 (95\% CI 1.43 to 1.65 ); women: HR 1.32 (95\% CI 1.23 to 1.43 ), table 3 ). Similarly, in patients with type $2 \mathrm{MI}$, the crude risk of MACE compared with those with type $1 \mathrm{MI}$ was also higher for both men and women, and was attenuated after adjustment but remained significant (men: HR 1.35 (95\% CI 1.26 to 1.44); women: HR 1.25 (95\% CI 1.17 to 1.35$)$ ).
In type $2 \mathrm{MI}$, a higher incidence rate of all-cause death and MACE was observed in men as compared with women (figure 3). Even after adjustment for risk factors and comorbidities, women were more likely to survive than men (HR 0.81 (95\% CI 0.74 to 0.90$)$, table 4). Differences in the risk of MACE between men and women were attenuated after adjustment for risk factors and comorbidities. Additional adjustment for coronary revascularisation and discharge medications in an explorative analysis yielded similar results (all-cause death: HR 0.82 (95\% CI 0.74 to 0.91 ); MACE: HR 0.92 (95\% CI0.84 to 1.01)).

We investigated the associations between clinical characteristics and outcomes in women and men with type 2 MI (table 5). A significant interaction for all-cause death was observed between sex and the risk factors of previous history of ischaemic stroke, previous history of CAD, hyperlipidaemia and hs-cTnT levels, and these risk factors seem more strongly associated with allcause death in women than in men. For MACE, a significant interaction with sex was observed for hyperlipidaemia and the previous history of CAD with a stronger relative importance of these conditions in women (online supplemental table S2).

\section{Secondary analyses}

We conducted secondary analyses by including patients with STEMI, and adjusting for the extent of CAD in the regression model. We observed that the results did not alter. When we included patients with STEMI in our analysis, both men and women with type 2 MI had a worse prognosis compared with patients with type $1 \mathrm{MI}$ (online supplemental table S3). Also men with type $2 \mathrm{MI}$ remained at increased risk of all-cause death compared with women with type 2 MI (online supplemental table S4). Our results also did not change when we adjusted for the extent of CAD (online supplemental tables S5-S6).

Table 3 Risk of all-cause death and major adverse cardiovascular events for patients with type 2 MI compared with type 1 MI stratified by sex

\begin{tabular}{|c|c|c|c|c|c|c|}
\hline & \multicolumn{2}{|l|}{ Total } & \multicolumn{2}{|l|}{ Men } & \multicolumn{2}{|l|}{ Women } \\
\hline & HR $(95 \% \mathrm{Cl})$ & $P$ value & HR $(95 \% \mathrm{Cl})$ & $P$ value & HR $(95 \% \mathrm{Cl})$ & $P$ value \\
\hline \multicolumn{7}{|l|}{ All-cause death* (type $1 \mathrm{Ml}$, ref cat) } \\
\hline Model 1 (crude) & 2.32 (2.20 to 2.44$)$ & $<0.001$ & 3.06 (2.85 to 3.28$)$ & $<0.001$ & $1.77(1.64$ to 1.91$)$ & $<0.001$ \\
\hline Model 2 (model 1+adjusted for comorbidities) & $1.44(1.36$ to 1.51$)$ & $<0.001$ & 1.54 (1.43 to 1.65$)$ & $<0.001$ & $1.32(1.23$ to 1.43$)$ & $<0.001$ \\
\hline Model 3 (model 2+adjusted for hs-cTnT concentrations) & 1.45 (1.38 to 1.53$)$ & $<0.001$ & 1.55 (1.44 to 1.67$)$ & $<0.001$ & $1.34(1.24$ to 1.45$)$ & $<0.001$ \\
\hline \multicolumn{7}{|l|}{ Major adverse cardiovascular eventst (type $1 \mathrm{MI}$, ref cat) } \\
\hline Model 1 (crude) & $2.00(1.91$ to 2.10$)$ & $<0.001$ & 2.49 (2.34 to 2.66$)$ & $<0.001$ & $1.62(1.51$ to 1.73$)$ & $<0.001$ \\
\hline Model 2 (model 1+adjusted for comorbidities) & 1.30 (1.24 to 1.36$)$ & $<0.001$ & 1.34 (1.25 to 1.43$)$ & $<0.001$ & 1.24 (1.16 to 1.33$)$ & $<0.001$ \\
\hline Model 3 (model 2+adjusted for hs-cTnT concentrations) & $1.31(1.25$ to 1.37$)$ & $<0.001$ & 1.35 (1.26 to 1.44$)$ & $<0.001$ & $1.25(1.17$ to 1.35$)$ & $<0.001$ \\
\hline
\end{tabular}

Model 1: MI type, sex, hospital site, year; model 2: model 1+age, current smoking, hypertension, diabetes, hyperlipidaemia, coronary artery disease, congestive heart failure, previous stroke, ST-segment depression, estimated glomerular filtration rate, COPD, dementia, previous or present cancer; model 3: model 2+hs-cTnT levels. In sex-stratified analysis, sex is not included in the models.

*Total, $n=47760$; women, $n=16$ 664; men, $n=31$ 096. $P_{\text {intercacion }}$ MI status $\times$ sex $=0.009$.

†Total, $n=45$ 863; women, $n=16$ 038; men, $n=29825$. Pintercion $M$ Ml status $\times$ sex $=0.152$.

COPD, chronic obstructive pulmonary disease; $h s-\mathrm{cTnT}$, hightion $\mathrm{sensitivity} \mathrm{cardiac} \mathrm{troponin} \mathrm{T}$; MI, myocardial infarction; ref cat, reference category. 


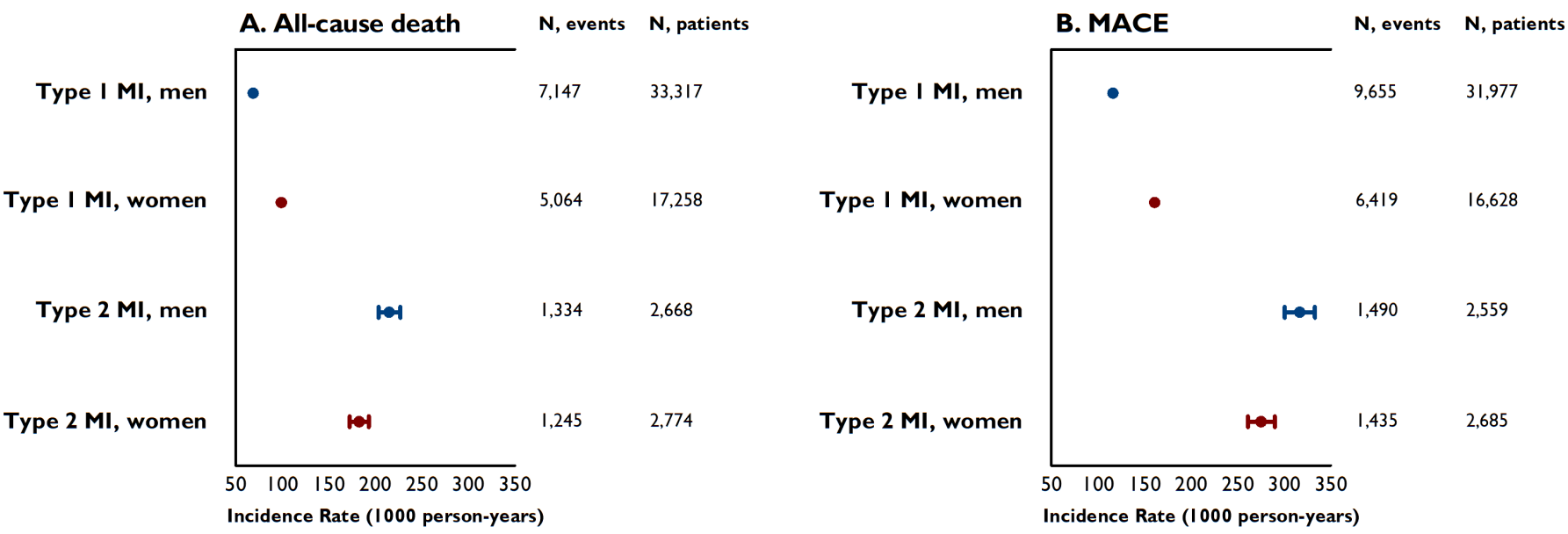

Figure 3 Sex-specific incidence rates (and their 95\% Cls) per 1000 person-years of all-cause death (panel A) and major adverse cardiovascular events (MACE) (panel B) for patients with type 1 and type 2 myocardial infarction (MI). MACE is defined as a composite end point of all-cause mortality, readmissions for non-fatal $\mathrm{MI}$, heart failure and ischaemic stroke.

\section{DISCUSSION}

We evaluated investigations and outcomes of patients admitted to a CCU in Sweden with type 1 and type 2 MI, stratified by sex. Our study has four main findings. First, while two-thirds of patients with type $1 \mathrm{MI}$ were men, the proportion of men and women with type 2 MI was identical. Second, although patients with type $2 \mathrm{MI}$ were less likely to undergo investigations than those with type 1 MI, there were no differences in the rates of echocardiography or CAG in type 2 MI by sex. More men than women had an impaired left ventricular (LV) function and obstructive CAD, and as a consequence women were less likely to undergo revascularisation. Third, men and women with type $2 \mathrm{MI}$ were at increased risk of death and MACE compared with those with type $1 \mathrm{MI}$, although these differences were attenuated after adjustment for baseline risk and comorbidities. Fourth, the prognosis of men with type 2 MI was worse than of women, even after adjustment for observed differences in baseline risk and comorbidities.

Our study has several strengths. The SWEDEHEART registry is a large database that consecutively collects data from patients with suspected acute coronary syndrome who are admitted to CCU in Sweden. This enabled us to gather data on $>60000$ patients with a diagnosis of $\mathrm{MI}$ and the largest series of patients with type 2 MI reported. The median follow-up of up to 2.8 years ensured enough outcome events to explore differences in determinants of prognosis between men and women.

Our data are in line with findings reported by Neumann et al observing similar proportions of men and women among patients

Table 4 Risk of all-cause death and major adverse cardiovascular events for men and women with type 2 myocardial infarction

\begin{tabular}{|c|c|c|}
\hline & $\mathrm{HR}(95 \% \mathrm{Cl})$ & $P$ value \\
\hline \multicolumn{3}{|l|}{ All-cause death ${ }^{*}$ (men, ref cat) } \\
\hline Model 1 (crude) & $0.82(0.75$ to 0.90$)$ & $<0.001$ \\
\hline Model 2 (model 1+adjusted for comorbidities) & $0.81(0.74$ to 0.90$)$ & $<0.001$ \\
\hline Model 3 (model $2+$ adjusted for hs-cTnT concentrations) & 0.84 (0.76 to 0.92$)$ & $\leq 0.001$ \\
\hline \multicolumn{3}{|l|}{ Major adverse cardiovascular eventst (men, ref cat) } \\
\hline Model 1 (crude) & $0.87(0.80$ to 0.95$)$ & 0.002 \\
\hline Model 2 (model 1 +adjusted for comorbidities) & $0.92(0.84$ to 1.01$)$ & 0.067 \\
\hline Model 3 (model $2+$ adjusted for hs-cTnT concentrations) & 0.94 (0.86 to 1.03$)$ & 0.189 \\
\hline \multicolumn{3}{|c|}{$\begin{array}{l}\text { Model 1: sex, hospital site, year; model 2: model 1+age, current smoking, hypertension, diabetes, hyperlipidaemia, } \\
\text { coronary artery disease, congestive heart failure, previous stroke, ST-segment depression, estimated glomerular } \\
\text { filtration rate, COPD, dementia, previous or present cancer; model } 3: \text { model } 2+\text { hs-cTnT levels. } \\
\text { *Patients with type } 2 \text { myocardial infarction, } n=4119 \text {. } \\
\text { tPatients with type } 2 \text { myocardial infarction } n=3979\end{array}$} \\
\hline
\end{tabular}

with type $2 \mathrm{MI}^{5}$ Our observations are also consistent with a study by McCarthy et al that included 359 patients with type 2 MI showing that men with type 2 MI had more cardiovascular morbidities than their female counterparts. ${ }^{15}$ Even though men from this cohort were younger than women, they presented with a greater burden of cardiovascular risk factors. In line with $\mathrm{McCa}-$ rthy et al, we found no differences in echocardiography and CAG between men and women, and observed a higher proportion of men with impaired LV function and obstructive CAD. McCarthy et al reported no difference on 90 days outcome. ${ }^{15}$ Conversely, in our prognostic analysis that included 5442 patients with type $2 \mathrm{MI}$, we showed that men with type $2 \mathrm{MI}$ had worse outcomes as compared with women. We believe that this is mostly explained by a greater burden of disease among men that increase cardiovascular risk. Nonetheless, the contribution of some risk indicators to adverse outcome was stronger in women as demonstrated by significant interaction terms. This indicates a greater relative importance of the absence/presence of these conditions in women compared with men. Accordingly, we speculate that men with type $2 \mathrm{MI}$ may be more likely to have pre-existent cardiovascular conditions contributing to the occurrence of type $2 \mathrm{MI}$ and the associated risks, while in women other factors contributing to myocardial oxygen supply/demand imbalance may predominate. Raphael et al recently showed that underlying mechanism of the supply/demand imbalance affects long-term outcome, and further investigation is needed to clarify whether the distribution of mechanisms differs by sex. ${ }^{16}$

Both men and women with type 2 MI have a poor prognosis. Our study showed that during the follow-up period 50\% and $45 \%$ of men and women, respectively, died following type $2 \mathrm{MI}$. Patients with type $2 \mathrm{MI}$ are at increased risk for future MACE as compared with patients having type 1 MI. ${ }^{12} 1718$ In line with our previous report, ${ }^{19}$ the risk of all-cause death and MACE was attenuated after adjustment of other risk factors in both men and women, suggesting that differences in clinical characteristics seem to partly explain the worse outcome in patients with type $2 \mathrm{MI}$.

A better understanding of the pathogenic mechanisms responsible for the different outcomes of men and women with type $2 \mathrm{MI}$ is urgently needed. Although coronary causes of myocardial oxygen supply-demand imbalance in type $2 \mathrm{MI}$, such as spontaneous coronary dissection, microvascular dysfunction or vasospasm, are less common than other cardiac or systemic causes, these conditions 
Table 5 Associations of clinical characteristics with all-cause death in type 2 myocardial infarction, stratified by sex

\begin{tabular}{|c|c|c|c|c|}
\hline & All & Men & Women & \\
\hline Clinical characteristics & $\mathrm{HR}(95 \% \mathrm{Cl})$ & HR $(95 \% \mathrm{Cl})$ & HR $(95 \% \mathrm{Cl})$ & $P_{\text {interaction }}$ with sex \\
\hline Sex (men, ref cat) & $0.83(0.76 \text { to } 0.92)^{*}$ & - & - & - \\
\hline Age (per 10 years) & $1.82(1.71 \text { to } 1.92)^{*}$ & 1.79 (1.65 to 1.94$)$ & 1.87 (1.72 to 2.04 ) & 0.615 \\
\hline Current smoking & $1.38(1.20 \text { to } 1.59)^{*}$ & 1.32 (1.09 to 1.60$)$ & 1.48 (1.20 to 1.82 ) & 0.359 \\
\hline Hypertension & $0.85(0.77 \text { to } 0.93)^{*}$ & 0.87 (0.76 to 0.99 ) & 0.80 (0.69 to 0.93 ) & 0.513 \\
\hline Diabetes & $1.28(1.15 \text { to } 1.42)^{\star}$ & 1.33 (1.15 to 1.53$)$ & 1.26 (1.08 to 1.47$)$ & 0.853 \\
\hline Hyperlipidaemia & $0.87(0.78 \text { to } 0.96)^{*}$ & $0.84(0.72$ to 0.97$)$ & 0.94 (0.81 to 1.10$)$ & $0.055 t$ \\
\hline Coronary artery disease & 1.10 (0.99 to 1.22$)$ & 0.96 (0.83 to 1.12$)$ & 1.26 (1.08 to 1.47$)$ & $0.002 t$ \\
\hline Congestive heart failure & $1.60(1.42 \text { to } 1.80)^{*}$ & 1.68 (1.43 to 1.96$)$ & 1.51 (1.26 to 1.82$)$ & 0.571 \\
\hline Ischaemic stroke & $1.44(1.27 \text { to } 1.63)^{*}$ & 1.31 (1.11 to 1.55$)$ & $1.58(1.32$ to 1.90$)$ & $0.067 \dagger$ \\
\hline ST-segment depression & $1.30(1.15 \text { to } 1.48)^{*}$ & 1.21 (1.02 to 1.44$)$ & 1.39 (1.16 to 1.66$)$ & 0.677 \\
\hline In eGFR & $0.62(0.57 \text { to } 0.68)^{*}$ & 0.64 (0.57 to 0.72 ) & 0.60 (0.52 to 0.69$)$ & 0.250 \\
\hline COPD & $1.71(1.54 \text { to } 1.92)^{*}$ & 1.58 (1.35 to 1.85$)$ & 1.87 (1.59 to 2.21$)$ & 0.143 \\
\hline Dementia & $1.53(1.01 \text { to } 2.34)^{*}$ & $1.33(0.71$ to 2.49$)$ & 1.61 (0.91 to 2.91$)$ & 0.749 \\
\hline Previous cancer & $1.45(1.24 \text { to } 1.70)^{*}$ & $1.40(1.16$ to 1.71$)$ & 1.61 (1.21 to 2.14$)$ & 0.283 \\
\hline In hs-cTnT & $1.14(1.09 \text { to } 1.18)^{*}$ & 1.11 (1.06 to 1.17$)$ & 1.18 (1.11 to 1.24$)$ & $0.077 t$ \\
\hline
\end{tabular}

are much more frequent in women. ${ }^{20} 21$ Similarly, there are wellestablished sex differences in type $1 \mathrm{MI}$ where women are more likely to have non-obstructive CAD and have better outcomes. ${ }^{22}$ A systematic evaluation of the role of CAD in men and women with type $2 \mathrm{MI}$ is needed. In those patients with type $2 \mathrm{MI}$ who underwent CAG here, obstructive CAD was observed in two-thirds of men and one-third of women. Despite this, significantly fewer men and women with type 2 MI underwent revascularisation compared with those with type $1 \mathrm{MI}$. This observation is perhaps not surprising and is consistent with other reports. ${ }^{23-25}$ While patients with type $2 \mathrm{MI}$ with CAD may benefit from revascularisation, there have been no randomised trials and such therapeutic approaches need evaluation in future research. The relationship between clinical characteristics and the diagnosis of type 2 MI also differed between men and women, with an interaction between sex and history of ischaemic stroke and CAD, hyperlipidaemia and hs-cTnT. These observations suggest that a sex-specific approach to management may be required in type $2 \mathrm{MI}$.

Several limitations of our analysis merit attention. First, the diagnosis of MI was based on discharge coding at the discretion of the treating physicians at each hospital. While the SWEDEHEART framework recommends the use of criteria outlined in the Universal Definition, ${ }^{12}$ there was no independent adjudication of the diagnosis which implies some risk of misclassification. This applies to the MI type $^{26}$ and to other conditions, for example, erroneous coding of Takotsubo syndrome as MI, especially during the first years of the study period when the awareness of this condition was limited. Nonetheless, our approach has the advantage of reflecting clinical practice. ${ }^{1427}$ Second, no data were available on the cause of type 2 MI. We lack information on the use of cardiac MRI that may have provided clarification in this regard. Third, only peak maximum cardiac troponin levels are documented in SWEDEHEART why we cannot comment on sampling frequencies or delta changes. Furthermore, we do acknowledge that we may limit generalisability by only including patients that were measured by the hs-cTnT assay. Fourth, the SWEDEHEART registry is restricted to patients who are admitted at CCU in Sweden. Patients with type 2 MI are often also admitted outwith the cardiology department, and MI management seems to differ with those who are treated at CCU. ${ }^{28}$ Extrapolating our findings to patients with MI managed in other facilities should thus be done with caution. Fifth, our composite end point of MACE

\section{Key messages}

What is already known on this subject?

- Type 2 myocardial infarction is a heterogenous condition with a poor prognosis.

- Whether this condition differs in men and women and how comorbidities and treatments influence outcome is unknown.

What might this study add?

- We report the clinical characteristics, investigations and outcomes in a large cohort of consecutive patients admitted to a coronary care unit in Sweden with type 1 and type 2 myocardial infarction, stratified by sex.

- Unlike in type 1 myocardial infarction, we observed that type 2 myocardial infarction occurred in men and women equally, and we found no evidence of sex bias in the selection of patients for cardiac investigations.

- Patients with type 2 myocardial infarction had worse outcomes, but women were less likely to have obstructive coronary artery disease or severe left ventricular impairment and were more likely to survive than men.

\section{How might this impact on clinical practice?}

- Given the overall poor prognosis, our findings highlight the need for meticulous evaluation and close follow-up in men and women with type 2 myocardial infarction.

- This should include consideration of the need for invasive or non-invasive coronary angiography, particularly in men given that obstructive disease was identified in two-thirds of those selected for testing.

- A better understanding of the underlying pathogenic mechanisms responsible for different outcomes of men and women with type 2 myocardial infarction is urgently needed, to inform prospective trials evaluating the potential for revascularisation and medical treatments to improve outcomes. 
did not capture re-infarction within 30 days, as the applied registry data do not enable us to distinguish a new MI from an index MI within that timeframe. Sixth, the majority of patients in the SWEDEHEART registry are Caucasian and extending our findings to other ethnic groups should be done with caution. Seventh, we did not consider patients with STEMI in the primary analysis as care pathways for this condition are highly standardised and the majority of patients have type 1 MI. Finally, we are unable to comment to which extent women with suspected MI are not referred to the CCU, and we cannot exclude systemic sex bias in the early management of patients with suspected acute coronary syndrome.

In summary, unlike in type $1 \mathrm{MI}$, we observed that type $2 \mathrm{MI}$ in patients admitted to Swedish CCUs occurred in men and women equally and we found no evidence of sex bias in the selection of patients for cardiac investigation. Patients with type $2 \mathrm{MI}$ had worse outcomes even after adjustment for differences in comorbidities, but women were less likely to have obstructive CAD or severe LV impairment and were more likely to survive than men.

\section{Twitter Andrew R Chapman @chapdoc1}

Contributors Concept and design: DMK, BL, NLM and KME. Data analysis and statistical analysis: DMK and KME. Manuscript draft: DMK, BL, NLM and KME. Critical revision, editing and approval of the final manuscript: all authors.

Funding The TOTAL-AMI project has received funding from the Swedish Foundation of Strategic Research. The study was supported by Health Data Research UK, which receives its funding from HDR UK Ltd (HDR-5012) funded by the UK Medical Research Council, Engineering and Physical Sciences Research Council, Economic and Social Research Council, Department of Health and Social Care (England), Chief Scientist Office of the Scottish Government Health and Social Care Directorates, Health and Social Care Research and Development Division (Welsh Government), Public Health Agency (Northern Ireland), British Heart Foundation and the Wellcome Trust. NLM is supported by the British Heart Foundation through a Senior Clinical Research Fellowship (FS/16/14/32023), Programme Grant (RG/20/10/34966) and a Research Excellence Award (RE/18/5/34216).

Competing interests SJRM received research funding and lecture fees from Abbott Laboratories and Roche Diagnostics. NLM reports research grants awarded to the University of Edinburgh from Abbott Diagnostics and Siemens Healthineers outside the submitted work, and honoraria from Abbott Diagnostics, Siemens Healthineers, Roche Diagnostics and LumiraDx. The other authors declared no conflicts of interest.

Patient consent for publication Not required.

Ethics approval The study was approved by the Ethical Review Board in Stockholm (2012/60-31/2) and conducted according to the principles of the 1975 Declaration of Helsinki.

Provenance and peer review Not commissioned; externally peer reviewed.

Data availability statement Data are available on reasonable request. The datasets generated during and/or analysed during the current study are not publicly available due to ethical restrictions and national laws, but are available on reasonable request under the provision that data may not leave Uppsala University.

Supplemental material This content has been supplied by the author(s). It has not been vetted by BMJ Publishing Group Limited (BMJ) and may not have been peer-reviewed. Any opinions or recommendations discussed are solely those of the author(s) and are not endorsed by BMJ. BMJ disclaims all liability and responsibility arising from any reliance placed on the content. Where the content includes any translated material, BMJ does not warrant the accuracy and reliability of the translations (including but not limited to local regulations, clinical guidelines, terminology, drug names and drug dosages), and is not responsible for any error and/or omissions arising from translation and adaptation or otherwise.

Open access This is an open access article distributed in accordance with the Creative Commons Attribution Non Commercial (CC BY-NC 4.0) license, which permits others to distribute, remix, adapt, build upon this work non-commercially, and license their derivative works on different terms, provided the original work is properly cited, appropriate credit is given, any changes made indicated, and the use is non-commercial. See: http://creativecommons.org/licenses/by-nc/4.0/.

\section{ORCID iDs}

Dorien M Kimenai http://orcid.org/0000-0003-1591-1352
Andrew R Chapman http://orcid.org/0000-0003-1926-5925

Nicholas L Mills http://orcid.org/0000-0003-0533-7991

\section{REFERENCES}

1 Chapman AR, Adamson PD, Shah ASV, et al. High-sensitivity cardiac troponin and the universal definition of myocardial infarction. Circulation 2020;141:161-71.

2 Chapman AR, Shah ASV, Lee KK, et al. Long-term outcomes in patients with type 2 myocardial infarction and myocardial injury. Circulation 2018;137:1236-45.

3 Thygesen K, Alpert JS, Jaffe AS, et al. Fourth universal definition of myocardial infarction (2018). J Am Coll Cardiol 2018;72:2231-64.

4 Defilippis AP, Chapman AR, Mills NL, et al. Assessment and treatment of patients with type 2 myocardial infarction and acute nonischemic myocardial injury. Circulation 2019;140:1661-78.

5 Neumann JT, Sörensen NA, Rübsamen N, et al. Discrimination of patients with type 2 myocardial infarction. Eur Heart J 2017;38:3514-20.

6 Sandoval Y, Jaffe AS. Type 2 myocardial infarction: JACC review topic of the week. J Am Coll Cardiol 2019:73:1846-60.

7 Sandoval Y, Smith SW, Sexter A, et al. Type 1 and 2 myocardial infarction and myocardial injury: clinical transition to high-sensitivity cardiac troponin I. Am J Med 2017;130:1431-9.

8 Lee KK, Ferry AV, Anand A, et al. Sex-specific thresholds of high-sensitivity troponin in patients with suspected acute coronary syndrome. J Am Coll Cardiol 2019:74:2032-43.

9 Mehta LS, Beckie TM, DeVon HA, et al. Acute myocardial infarction in women: a scientific statement from the American heart association. Circulation 2016;133:916-47.

10 Wenger NK. Women and coronary heart disease: a century after Herrick: understudied, underdiagnosed, and undertreated. Circulation 2012;126:604-11.

11 Jernberg T, Attebring MF, Hambraeus K, et al. The Swedish Web-system for enhancement and development of evidence-based care in heart disease evaluated according to recommended therapies (SWEDEHEART). Heart 2010;96:1617-21.

12 SWEDEHEART. Infarktdefinition_140515 KE (2). Available: http://www.ucr.uu.se/ swedeheart/dokument-rikshia/ovriga-dokument-rikshia [Accessed 30 Jan 2021].

13 von Elm E, Altman DG, Egger M, et al. The strengthening the reporting of observational studies in epidemiology (STROBE) statement: guidelines for reporting observational studies. Lancet 2007;370:1453-7.

14 Ludvigsson JF, Andersson E, Ekbom A, et al. External review and validation of the Swedish national inpatient register. BMC Public Health 2011;11:450.

15 McCarthy CP, Murphy S, Cohen JA, et al. Sex differences in patient characteristics, treatment strategies, and outcomes for type 2 myocardial infarction. J Am Coll Cardiol 2019;73:3230-2.

16 Raphael CE, Roger VL, Sandoval Y, et al. Incidence, trends, and outcomes of type 2 myocardial infarction in a community cohort. Circulation 2020;141:454-63.

17 Shah ASV, McAllister DA, Mills R, et al. Sensitive troponin assay and the classification of myocardial infarction. Am J Med 2015;128:493-501.

18 Smilowitz NR, Subramanyam P, Gianos E, et al. Treatment and outcomes of type 2 myocardial infarction and myocardial injury compared with type 1 myocardial infarction. Coron Artery Dis 2018;29:46-52.

19 Baron T, Hambraeus K, Sundström J, et al. Type 2 myocardial infarction in clinical practice. Heart 2015;101:101-6.

20 Smilowitz NR, Mahajan AM, Roe MT, et al. Mortality of myocardial infarction by sex age, and obstructive coronary artery disease status in the action Registry-GWTG (acute coronary treatment and intervention outcomes network Registry-Get with the guidelines). Circ Cardiovasc Qual Outcomes 2017:10:e003443.

21 Agewall S, Beltrame JF, Reynolds HR, et al. Esc Working group position paper on myocardial infarction with non-obstructive coronary arteries. Eur Heart J 2017:38:143-53.

22 Tamis-Holland JE, Jneid H, Reynolds HR, et al. Contemporary diagnosis and management of patients with myocardial infarction in the absence of obstructive coronary artery disease: a scientific statement from the American heart association. Circulation 2019;139:e891-908.

23 Cediel G, Gonzalez-Del-Hoyo M, Carrasquer A, et al. Outcomes with type 2 myocardia infarction compared with non-ischaemic myocardial injury. Heart 2017:103:616-22.

24 Saaby L, Poulsen TS, Hosbond S, et al. Classification of myocardial infarction: frequency and features of type 2 myocardial infarction. Am J Med 2013;126:789-97.

25 Stein GY, Herscovici G, Korenfeld R, et al. Type-II myocardial infarction--patient characteristics, management and outcomes. PLoS One 2014;9:e84285.

26 Gard A, Lindahl B, Batra G, et al. Diagnosing type 2 myocardial infarction in clinical routine. A validation study. Scand Cardiovasc J 2019;53:259-65.

27 Gard A, Lindahl B, Batra G, et al. Interphysician agreement on subclassification of myocardial infarction. Heart 2018;104:1284-91.

28 Gard A, Lindahl B, Hadziosmanovic N, et al. Treatment and prognosis of myocardial infarction outside cardiology departments. J Clin Med 2020;10:106-3. doi:10.3390/ jcm10010106 